\title{
TENDENCIES TO REPEATING OF TYPE-IVm BURSTS AND THEIR RELATIONS TO THE STAGE OF DEVELOPMENT OF THE SUNSPOT GROUP
}

\author{
A. BöHME* \\ (Heinrich-Hertz-Institute of Solar Terrestrial Physics, Berlin-Adlershof, D.D.R.)
}

\begin{abstract}
A BSTRACT
It has been shown that moving type-IV bursts at decameter waves are infrequent events compared with the moving type-IV bursts at meter waves. They belong preferably to sunspot groups of relatively homogeneous magnetic structure. Moving type-IV bursts at decameter waves have a very low tendency to repeat. This statement indicates that the magnetic structures producing them have only a short lifetime. The short duration of these bursts cannot be interpreted by radiation damping of high energetic electrons. Furthermore it can be shown that the storm continuum at decameter waves is related preferably to sunspot groups of complex magnetic structure. Probably stationary type-IV bursts at decameter waves are more representative for the acceleration of high-energetic particles than the moving type-IV bursts at decameter waves.
\end{abstract}

In a recent study Antalova (1967) has shown that type-IV bursts originate only during certain stages of development of the associated sunspot groups. She classified seven different spot-group configurations in the photosphere, which can produce type-IV bursts. Now it was our aim to find out whether some of the sub-types of the type-IV burst are related to special configurations of the sunspot groups. In the present investigation the type-IV bursts were taken from the catalogues of PickGutmann, Švestka-Olmr and Fokker, the Quarterly Bulletin, and from the measurements of the Heinrich-Hertz-Institute. The classification of the sunspot groups was based upon the Antalova scheme for which drawings were used obtained by the Potsdam Solar Observatory. The distribution of the type-IV bursts on the various configurations obtained by us agrees well with the distribution given by Antalova.

The first sub-type under consideration was the moving type-IV burst at $40 \mathrm{MHz}$ and lower frequencies. From interferometric observations made by Weiss (1963) it can be seen that at $40 \mathrm{MHz}$ a moving type-IV burst is a rather infrequent event compared with the frequency range between 170 and $100 \mathrm{MHz}$. There is also indirect evidence for this from the observations illustrated by Figure 1, where the number of the type-IV bursts we have measured at 111 and $40 \mathrm{MHz}$, together with measurements of the National Bureau of Standards (Boulder) at 108 and $18 \mathrm{MHz}$, are plotted

\footnotetext{
* Presented by A. Krüger.
} 


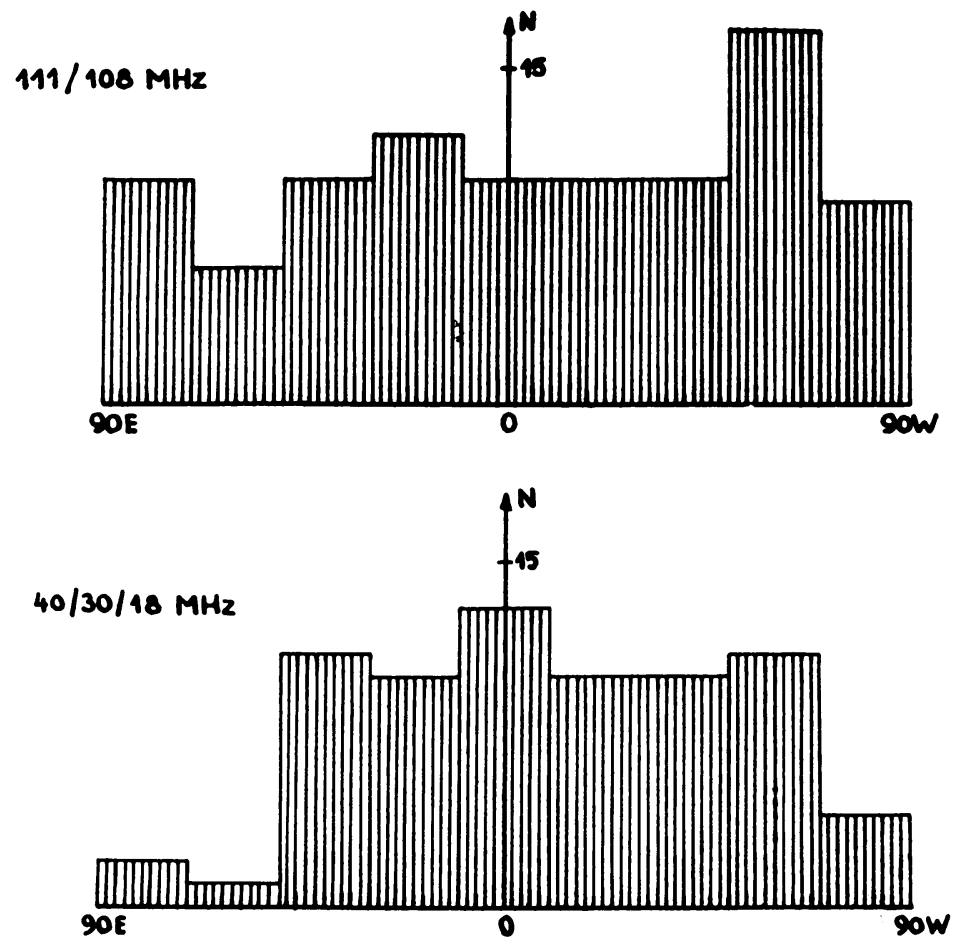

FIG. 1. Center-limb variation of the number of type-IV bursts at $111 / 108$ and $40 / 30 / 18 \mathrm{MHz}$.

in dependence of the distance from the central meridian on the Sun. While there are at $111 / 108 \mathrm{MHz}$ hardly any center-limb variations, the number of bursts in the decameter wave region decreases remarkably towards the limb, which suggests the absence of a moving component.

Though we had no interferometric measurements available, the attempt was made to find out whether or not the type-IV bursts observed at our institute have a moving component at 40, 30, and $23 \mathrm{MHz}$. Fortunately, since 1961 polarization observations in the decameter-wave region were available and we assigned a moving component to those bursts which exhibited either a reversal of the sense of the polarization between a first part and a second part, or which - with the flare near to the limb showed, in accordance with the leading-spot hypothesis, an extraordinary polarization. Based on these experiences we have assigned a moving component also to the type-IV bursts measured prior to 1961 , if at the beginning there was a continuum with a frequency drift of the order of some minutes, a duration greater than 5 and smaller than $25 \mathrm{~min}$, corresponding to the flash phase of the centimeter bursts.

Together with the cases reported by Weiss and Philip (1964) material on 33 moving type-IV bursts in the decameter-wave region was available. These bursts have some 
properties obviously differing from other type-IV bursts. In general, type-IV bursts have the tendency of occurring in active centres repeatedly. Only $26 \%$ of all the type-IV bursts occurred as single events in an active centre. But 21 out of the 33 moving type-IV bursts in the decameter-wave region belonged to single events in the respective active centre. Only 2 out of the remaining 12 type-IV bursts occurred repeatedly in the same centre. This rather weak tendency for recurrence indicates that moving type-IV bursts in the decameter-wave region are obviously associated with rather short-lived magnetic-field configurations.

This assumption is also supported by consideration of Figure 2. In the first line can be seen the distribution of all type-IV bursts to the group named 'complex', to the group $\mathrm{H}^{\prime}$ and to the other groups given by Antalova. It can be seen that, as compared with all type-IV bursts, only a conspicuously small fraction of the moving type-IV bursts in the decameter-wave region belongs to the group named 'complex'.

$\begin{array}{lccccc} & \begin{array}{c}\text { Total } \\ \text { number } \\ \text { of bursts }\end{array} & \text { Complex } & \mathrm{H}^{\prime} & \begin{array}{c}\text { HE, E', R, } \\ \mathrm{F}^{\prime}, 2 \mathrm{~F}, \mathrm{D}\end{array} & \text { Unclassified } \\ \begin{array}{l}\text { All type-IV bursts } \\ \begin{array}{l}\text { Moving type-IV bursts } \\ \text { at } 40 \mathrm{MHz}\end{array}\end{array} & 198 & 43 \% & 27 \% & 18 \% & 12 \% \\ \begin{array}{l}\text { Type-IV bursts without } \\ \text { stationary component } \\ \text { at } 40 \mathrm{MHz}\end{array} & 33 & 27 & 37 & 36 & - \\ \begin{array}{l}\text { Weak stationary type-IV } \\ \text { bursts at 40 MHz }\end{array} & 33 & 42 & 33 & 25 & - \\ \begin{array}{l}\text { Storm continua at } 40 \mathrm{MHz} \\ \text { and lower frequencies }\end{array} & 27 & 41 & 37 & 15 & 7 \\ \end{array}$

FIG. 2. Distribution of type-IV bursts at the different stages of development of the related spot groups.

On the other hand, $70 \%$ of the type-IV bursts which have no moving component in the decameter-wave region belong to the group named 'complex'. This observation suggests that obviously the ejection of a plasma cloud with the great speed observed for moving type-IV bursts in the decameter-wave region occurs mainly in not very inhomogeneous magnetic fields.

After Warwick and Wood Haurwitz (1962) type-IV bursts in the decameter-wave region are especially typical for the production of high-energy particles in the active centre. In order to check if the short duration observed by Weiss and Philip of the moving type-IV bursts in the decameter-wave region could be explained by the radiation damping of high-energy electrons, we compared the maximum intensities at $3000 \mathrm{MHz}$ of the type-IV bursts with and without a moving component in the decameter-wave region, because the maximum intensity in the centimeter region can also be regarded as an indication for the existence of high-energy particles. It was 
then, however, found that the intensity of type-IV bursts with a moving component in the decameter-wave region at $3000 \mathrm{MHz}$, on the average, is less than that of the type-IV bursts without a moving component. This suggests that the short duration of the moving type-IV bursts cannot be explained by radiation damping and that, further, the high ejection speeds are caused by magnetic-field configurations rather than by high-energy production in the flare.

In addition to the moving type-IV bursts there has been investigated also the stationary type-IV burst in the decameter-wave region. As is recognizable from Figure 2, the storm continua with a component at $40 \mathrm{MHz}$ and less are associated

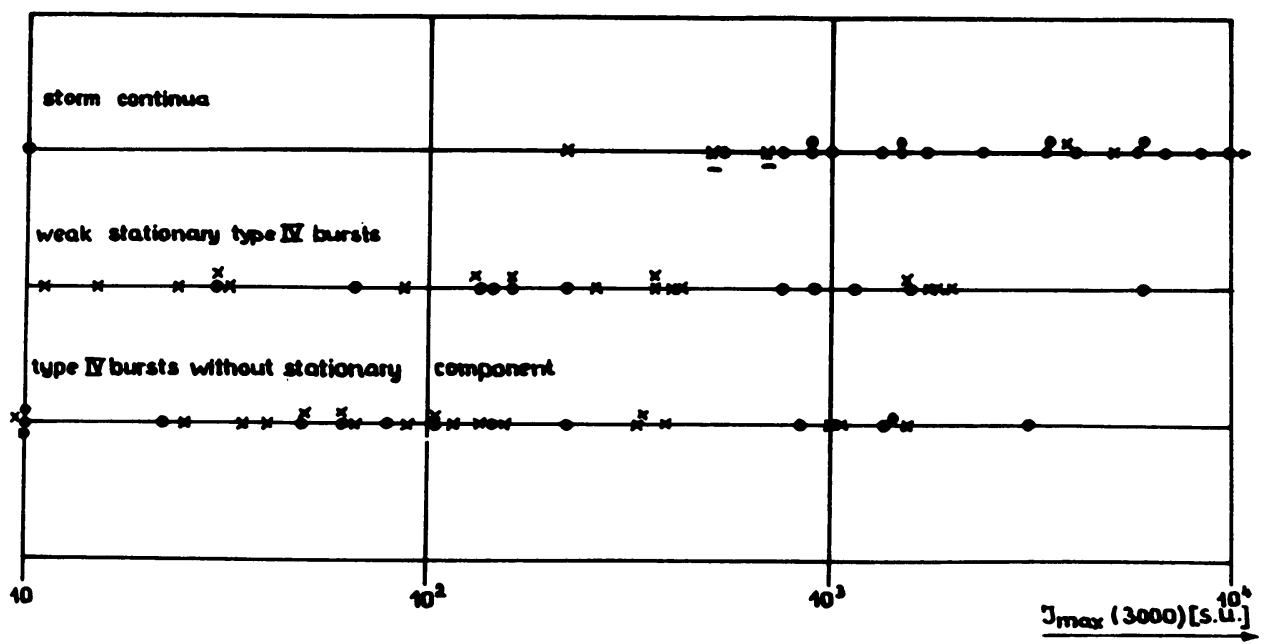

- Complex

$\times$ H...D

FIG. 3. The distribution of $I_{\max }(3000)$ for type-IV bursts with and without stationary component at $40 \mathrm{MHz}$.

in $86 \%$ of the cases with spots of the class 'complex'. This value is far in excess of the average of the total of the type-IV bursts. Obviously, the configurations producing storm continua are very stable. Nearly all the active centres, which for one or two solar rotations have produced type-IV bursts, have emitted during their formation one or more storm continua with a component in the decameter-wave region. It is, further, of some interest that the only two storm continua which had no component in the decameter-wave region belonged to the spot groups of class $\mathrm{H}^{\prime}$ or $\mathrm{R}$, respectively.

Only $41 \%$ of the centres which only produced stationary type-IV bursts of less than 2 hours' duration and low intensity belonged to the class named 'complex'. As can be seen from Figure 3 a distinction between storm continua and stationary type-IV bursts of low intensity in the decameter-wave region is indicated by the intensity of 
the component in the cm-wave region. The figure contains the distribution of $I_{\max }(3000)$ for storm continua, weak stationary bursts, and type-IV bursts without a stationary component in the decameter-wave region. It can be seen that storm continua in the decameter-wave region preferably have intense components at $3000 \mathrm{MHz}$. Hence, it follows that just the strong stationary bursts and not the moving type-IV bursts in the decameter-wave region are the ones typical for the production of highenergy particles, in spite of the fact that stationary bursts are likely to be interpreted as Cerenkov radiation and, hence, not expected to be produced by particles with energies in the $\mathrm{MeV}$ region.

In conclusion it can be said that the type-IV bursts, which have no stationary component in the meter- and decameter-wave region are obviously typical for a very short-lived state of the magnetic field in the spot groups during which, probably, no large amount of energy is released. Considering flares at distances smaller than $60^{\circ}$ from the central meridian of the Sun there was no case when a subsequent occurrence of two type-IV bursts without stationary component was observed in one active centre. In the majority of cases after a simple moving type-IV burst the centre of activity was unable to continue emitting further type-IV bursts. In the rather infrequent cases of type-IV bursts following a simple moving type-IV burst in the same centre of activity, these bursts always have again stationary components, which suggests that the magnetic field has changed its configuration.

\section{References}

Antalova, A. (1967) Bull. astr. Inst. Csl., 18, 61.

Philip, K.W. (1964) Astrophys. J., 139, 723.

Warwick, C.S., Wood Haurwitz, M.J.(1962) J. geophys. Res., 67, 1317.

Weiss, A. A. (1963) Austr.J. Phys., 16, 526. 